\title{
Reply to: Li J, Cao Y "Serum ferritin as a biomarker for diabetes and insulin resistance: a further study"
}

\author{
Yiqiang Zhan $\cdot$ Jinming Yu
}

Received: 28 January 2015 / Accepted: 5 February 2015/Published online: 28 February 2015

(C) Springer-Verlag Italia 2015

We thank Drs. Li and Cao for their careful comments [1] to our recent report regarding the association between serum ferritin and diabetes [2]. We agree with the point that metabolic syndrome (MS) components should be taken into account of the analysis or adjusted for as confounders in the multiple regression models. In our paper, we have already adjusted for several metabolic biomarkers including BMI, serum lipids, and hypertension. Additional adjustment for other MS components, such as waist circumference, might incur over adjustment due to collinearity.

As for excluding the anemic participants, we cannot find strong evidence to back this up. Firstly, anemia's association with diabetes has not been well established, although its association with ferritin is obvious. Thus, anemia (or hemoglobin) does not fulfill the current definition of confounding and should not be adjusted for or excluded [3]. Secondly, when anemic participants were excluded, serum ferritin was still associated with higher odds of diabetes as shown in Table 1 in Dr Li's comments, even though the association did not reach a statistically significant level. We still cannot exclude the possibility that Dr Li's further analysis might be a false negative result

Managed by Antonio Secchi.

Y. Zhan $(\bowtie) \cdot$ J. Yu

Institute of Clinical Epidemiology, School of Public Health,

Fudan University, 130 Dong'An Road, Shanghai 200032,

People's Republic of China

e-mail: zhany09@fudan.edu.cn

J. $\mathrm{Yu}$

e-mail: jmy@fudan.edu.cn

Y. Zhan · J. Yu

Key Laboratory of Public Health Safety, School of Public

Health, Fudan University, Shanghai, China and it might become statistically significant when analyzed in a larger sample. The argument that Dr Li's further study was inconsistent with our results might be too strong. Lastly, because both of our analyses were based on a crosssectional survey, no causal relationship can be inferred due to the natural drawbacks of the cross-sectional design. Further longitudinal studies with repeated measurements of anemia, ferritin, and diabetes should be of paramount value to disentangle the causality among them.

Conflict of interest Yiqiang Zhan and Jinming Yu declare that they have no conflict of interest.

Ethical standard This article does not contain any studies with human or animal subjects performed by any of the authors.

Informed consent This study does not involve human or animal subjects. No informed consent needs to be obtained.

\section{References}

1. Li J, Cao Y (2015) Serum ferritin as a biomarker for diabetes and insulin resistance: a further study. Acta Diabetol. doi:10.1007/ s00592-015-0719-y

2. Zhan Y, Tang Z, Yu J (2014) Serum ferritin, diabetes, diabetes control, and insulin resistance. Acta Diabetol 51:991-998

3. Greenland S, Pearl J, Robins JM (1999) Causal diagrams for epidemiologic research. Epidemiology 1:37-48 